\title{
Predictors of Central Compartment Involvement in Patients with Positive Lateral Cervical Lymph Nodes According to Clinical and/or Ultrasound Evaluation
}

\author{
Giuseppa Graceffa ${ }^{1}$, Giuseppina Orlando ${ }^{2, *}$, Gianfranco Cocorullo ${ }^{2}$, Sergio Mazzola ${ }^{3}$, Irene Vitale ${ }^{2}$, \\ Maria Pia Proclamà ${ }^{2}$, Calogera Amato ${ }^{2}$, Federica Saputo ${ }^{1}$, Enza Maria Rollo ${ }^{1}$, Alessandro Corigliano ${ }^{4}$, \\ Giuseppina Melfa $^{2} \mathbb{D}$, Calogero Cipolla ${ }^{1}$ and Gregorio Scerrino ${ }^{4}$
}

1 Unit of Oncological Surgery, Department of Surgical Oncological and Oral Sciences, University of Palermo, Via del Vespro, 129, 90127 Palermo, Italy; giuseppa.graceffa@unipa.it (G.G.); federica.saputo92@gmail.com (F.S.); enzamaria13@gmail.com (E.M.R.); calogero.cipolla@unipa.it (C.C.)

2 Unit of General and Emergency Surgery, Department of Surgical Oncological and Oral Sciences, Policlinico P. Giaccone, University of Palermo, Via L Giuffré, 5, 90127 Palermo, Italy; gianfranco.cocorullo@unipa.it (G.C.); irenevitale93@gmail.com (I.V.); mariapiaproclama@gmail.com (M.P.P.); calogera.amato92@gmail.com (C.A.); irene_melfa@yahoo.it (G.M.)

3 Unit of Clinical Epidemiology \& Tumor Registry, Department of Laboratory Diagnostics, Policlinico P. Giaccone, University of Palermo, Via L Giuffré, 5, 90127 Palermo, Italy; mazzolasergio@hotmail.it

4 Unit of Endocrine Surgery, Department of Surgical Oncological and Oral Sciences, Policlinico P. Giaccone, University of Palermo, Via L Giuffré, 5, 90127 Palermo, Italy; alessandro-corigliano@hotmail.it (A.C.);

Citation: Graceffa, G.; Orlando, G.; Cocorullo, G.; Mazzola, S.; Vitale, I.; Proclamà, M.P.; Amato, C.; Saputo, F.; Rollo, E.M.; Corigliano, A.; et al. Predictors of Central Compartment Involvement in Patients with Positive Lateral Cervical Lymph Nodes According to Clinical and/or Ultrasound Evaluation. J. Clin. Med. 2021, 10, 3407. https://doi.org/ $10.3390 / \mathrm{jcm} 10153407$

Academic Editor: Giovanni Conzo

Received: 14 July 2021

Accepted: 29 July 2021

Published: 30 July 2021

Publisher's Note: MDPI stays neutral with regard to jurisdictional claims in published maps and institutional affiliations.

* Correspondence: giusi_orlando@hotmail.it

\begin{abstract}
Lymph node neck metastases are frequent in papillary thyroid carcinoma (PTC). Current guidelines state, on a weak level of evidence, that level VI dissection is mandatory in the presence of latero-cervical metastases. The aim of our study is to evaluate predictive factors for the absence of level VI involvement despite the presence of metastases to the lateral cervical stations in PTC. Eightyeight patients operated for PTC with level II-V metastases were retrospectively enrolled in the study. Demographics, thyroid function, autoimmunity, nodule size and site, cancer variant, multifocality, Bethesda and EU-TIRADS, number of central and lateral lymph nodes removed, number of positive lymph nodes and outcome were recorded. At univariate analysis, PTC location and number of positive lateral lymph nodes were risk criteria for failure to cure. ROC curves demonstrated the association of the number of positive lateral lymph nodes and failure to cure. On multivariate analysis, the protective factors were PTC located in lobe center and number of positive lateral lymph nodes $<4$. Kaplan-Meier curves confirmed the absence of central lymph nodes as a positive prognostic factor. In the selected cases, Central Neck Dissection (CND) could be avoided even in the presence of positive Lateralcervical Lymph Nodes (LLN+).
\end{abstract}

Keywords: papillary thyroid carcinoma; central compartment; lateralcervical lymph nodes; EUTIRADS; Bethesda; central neck dissection; lateral neck dissection; skip metastasis

\section{Introduction}

Papillary thyroid carcinoma (PTC) has an extremely strong tendency to metastasize to the neck lymph nodes. This condition can occur in up to $80 \%$ of cases $[1,2]$. There is widespread acceptance of the classification of neck lymph nodes into seven levels, with level VI being the lymph nodes of the central compartment and level VII the lymph nodes of the upper mediastinum. Level I (sub-mental and sub-mandibular lymph nodes) is generally not considered in PTC exeresis, whereas levels II to V are the lymph nodes of the lateral compartment involved in neck dissection [3].

The current ATA guidelines (2015) regarding prophylactic central neck dissection (PCND) limit this indication to stages from T3 (tumor $>4 \mathrm{~cm}$ in greatest diameter and/or 
gross invasion of prethyroid muscles), while therapeutic central neck dissection is indicated in T1 (tumor less than $2 \mathrm{~cm}$ ) or T2 (tumor size between 2 and $4 \mathrm{~cm}$ ) only in the clear presence (clinically, ecographically and/or biopsy proven) of central metastases, or in cases of lateral lymphadenopathy [4-6]. Although widely accepted and practiced, this indication, contained in recommendation 36 of the ATA guidelines, is weak, with a low level of evidence [4].

Central neck dissection is not a procedure without complications. Hypoparathyroidism and recurrent laryngeal nerve palsy (both transient or permanent) may occur more frequently than in simple thyroidectomy [7-12].

The realization that central neck dissection is a surgical procedure with additional morbidity may lead to the search for a context in which, even in the presence of metastases to the lateral neck lymph nodes, central neck dissection could be avoided. The occurrence of skip metastasis to the lateral lymph node compartment is well known [13-15]. However, only recently a study appeared in the literature specifically aimed at answering the question of whether prophylactic central compartment dissection is always needed in the presence of lateral neck metastases [16].

The aim of this study is to evaluate predictive factors for the absence of level VI involvement despite the presence of metastases to the lateral cervical stations in PTC, and also to formulate hypotheses on the possible risk of persistence or recurrence directly related to the persistence of level VI lymph nodes.

\section{Material and Methods}

This retrospective observational cohort study was carried out on patients consecutively that had undergone thyroidectomy from January 2010 to December 2020, with the diagnosis of papillary thyroid carcinoma at two University Surgery Units: the General and Emergency Surgery Unit and the General and Oncology Surgery, both of which are referral centers for endocrine neck surgery in western Sicily. All patients with complete clinical reports regarding preparation for surgery, hospital course and follow-up until 30 June 2021 were included in the study; patients included underwent surgery for PTCs with metastases to the lateral-cervical lymph nodes at the time of surgery and who, therefore, underwent TT + CND + unilateral LND in one step. Surgical procedures were performed by surgeons, belonging to the respective operating units, classifiable as "high volume" according to the unanimous consensus of the international literature $[17,18]$, having all performed over 1000 thyroidectomies with more than 100 procedures/year for a period of activity of 10 or more years.

Exclusion criteria were: surgery for central and/or latero-cervical metastases, which, therefore, underwent Thyroidectomy and/or Central Neck Dissection and/or Lateral Neck Dissection in two or more steps. We also excluded non-papillary thyroid cancers, patients with incomplete clinical documentation, those with malignancy of another site or who had developed an extrathyroid cancer during follow-up, familial thyroid tumors and who had undergone operations performed by operators with a volume of activity of less than 1000 total thyroidectomies or $<100$ thyroidectomies / year or with a period of "dedicated" activity of less than 10 years.

We considered the following variables (in brackets, the way the variables were assessed): age (continuous), sex (categorical), TSH values detected at the time of preparation for surgery (continuous), autoimmunity (categorical), largest diameter of the nodule measured at ultrasound (continuous), cancer variant (classical, follicular, other, categorical). Based on preoperative ultrasonographic findings, we included the suspicious lesions in the excel sheet classifying them as located in the "upper lobe pole" (=1), "middle lobe" (=2) and "other" (=3), including in this group the isthmic or paraisthmic lesions and the lesions at the lower pole of the thyroid lobe (categorical variable). Moreover, we recorded multifocality (categorical), Bethesda classification (categorical), EU-TIRADS classification (categorical), total number of central lymph nodes removed (continuous), number of positive central lymph nodes (continuous), total number of lateral lymph nodes removed (continuous), 
number of positive lateral lymph nodes (continuous) and outcome (unfavorable, yes/no, categorical). The categories that we considered as "unfavorable" outcomes fell into two different patterns: patients with "persistence", in whom there was proven locoregional disease before six months after postoperative radioiodine ablation, and patients with "recurrence", in whom new disease occurred after this cut-off time. The presence of locoregional disease (persistence or recurrence) was assessed by integrating the results of laboratory tests (thyroglobulin $[\mathrm{Tg}]>10 \mathrm{ng} / \mathrm{mL}$ after appropriate discontinuation of L-Tyroxine treatment in the absence of anti-Tg antibodies), ultrasound (presence of suspected locoregional tissue) and fine-needle aspiration biopsy of tissue detected on ultrasound, which in turn was evaluated by cytology and $\mathrm{Tg}$ assay on the eluate. In two cases, there was persistence of elevated $\mathrm{Tg}$ values in the absence of locoregional recurrence on instrumental exams: these patients were excluded from the study because of the uncertainty of considering them as loco-regional recurrence, systemic recurrence or false positive result.

For the purpose of the Kaplan-Meier curves, the date on which the recurrence was detected was reported. Disease-free patients were conventionally verified as of 30 June 2021.

Among the variables included in the statistical evaluation, location within the thyroid lobe was documented in the literature as a risk factor for skip metastasis [13-15], while variables such as the threshold value of metastatic lateral lymph nodes and the total number of central lymph nodes removed were derived from the study results. TIRADS and cytology (classified according to Bethesda) were included in order to evaluate whether these two simple datapoints from the preoperative diagnostic procedure could be used as indicators and guide the choice of surgery.

\subsection{Surgery}

The surgical procedure has always been performed as a 'formally' total thyroidectomy. In the patients undergoing Intraoperative Nerve Monitoring, if the surgical protocol required to suspend the operation at lobectomy with removal of the central hemicompartment ipsilateral to the cancer (two-staged thyroidectomy), we excluded these patients (two in the whole cohort) from the study. Central neck dissection was always bilateral, with excision of both periricurrential and paratracheal chains, and lateral neck dissection extended at least to IIa-III-IV levels, while Ilb and V levels were removed in the presence of gross and/or multiple latero-cervical metastases detected by ultrasound or in the presence of involvement of these stations.

\subsection{Radioiodine Ablation and Endocrinological Treatment}

All patients with metastatic lateral lymph node (LLN+) underwent radioiodine ablation, at a dosage of about 100-150 mCi, after suspension of L-thyroxine treatment until TSH values $>30 \mathrm{microIU} / \mathrm{mL}$ were reached. This treatment was performed 4-8 weeks after surgery and, after post-treatment whole-body scan, suppressive therapy with L-thyroxine was reimposed with the aim of bringing TSH to about $0.5 \mathrm{microIU} / \mathrm{mL}$.

\subsection{Statistical Analysis}

In a first step, a univariate analysis was carried out in which Fisher's exact test for categorical variables and Mann-Whitney's test for continuous variables were applied.

ROC curves were then realized in order to evaluate the accuracy of the variables "number of central lymph node (CLN)", "number of lateral lymph node (LLN)" and "number of LLN+" as predictors of the occurrence of metastatic central lymph node $(\mathrm{CLN}+\geq 1)$.

Moreover, a multivariate logistic analysis was performed in which the following variables were included: Age, Sex, TIRADS, Location, CLN and LLN+. From the various models, the best fit was chosen. Akaike's Information Criterion (AIC) was used as an indicator of the quality of the fit of the multiple logistic regression function to the needs of the study. 
Finally, Kaplan-Meier curves were created in the following three groups: CLN+ = 0, All cases and CLN $+\geq 1$. The statistical significance of the differences between the KaplanMeier curves was checked with the Log Rank test.

Statistical elaborations were carried out with the software RStudio (version 3.4.1 of 30 June 2017) for R (version 2.1) The ROC Curves and Kaplan-Meier were made using the application packages "pROC" and "Survival".

\section{Results}

Eighty-eight patients in follow-up from 6 months to 10 years (mean 4.6 years) with a mean age of 46 years (range: 14-82) met the inclusion criteria.

\subsection{Univariate Analysis}

From the univariate analysis (Table 1 ) the variables with $p$-value $<0.05$ were: Location, TIRADS, CLN and LLN+. The location, TIRADS and LLN+ seem to be a moderately accurate predictor of $\mathrm{CLN}+$. Comparing the ROC curves, in which the specificity/sensitivity relationship was calculated using CLN+ as a reference we note that the number of LLN is a poor predictor of CLN+ (Figure 1). In fact, the Area Under the Curve (AUC) values were $=0.57$ and $95 \%$ confidence interval $(\mathrm{CI})(0.44-0.68)$, with a threshold value of 28.5. Specificity, therefore, was $1 \% \mathrm{CI}(1-1)$ and sensitivity $=0.18$ with $95 \% \mathrm{CI}(0.09-0.29)$. The numbers of CLN and LLN+ were found to be a moderately accurate predictor of CLN+ with $\mathrm{AUC}=0.73$ and $95 \% \mathrm{CI}(0.61-0.82)$ with a cutoff value $=7.5$, specificity $=0$. $84 \% \mathrm{CI}(0.72-0.97)$ and sensitivity $=0.46$ with $95 \% \mathrm{CI}(0.34-0.59)$ and AUC $=0.83$ and $95 \%$ CI $(0.73-0.92)$ with a cutoff value of 2.5 , specificity $=0.81 \% \mathrm{CI}(0.66-0.94)$ and sensitivity $=0.77$ with $95 \%$ CI $(0.66-0.88)$, respectively.

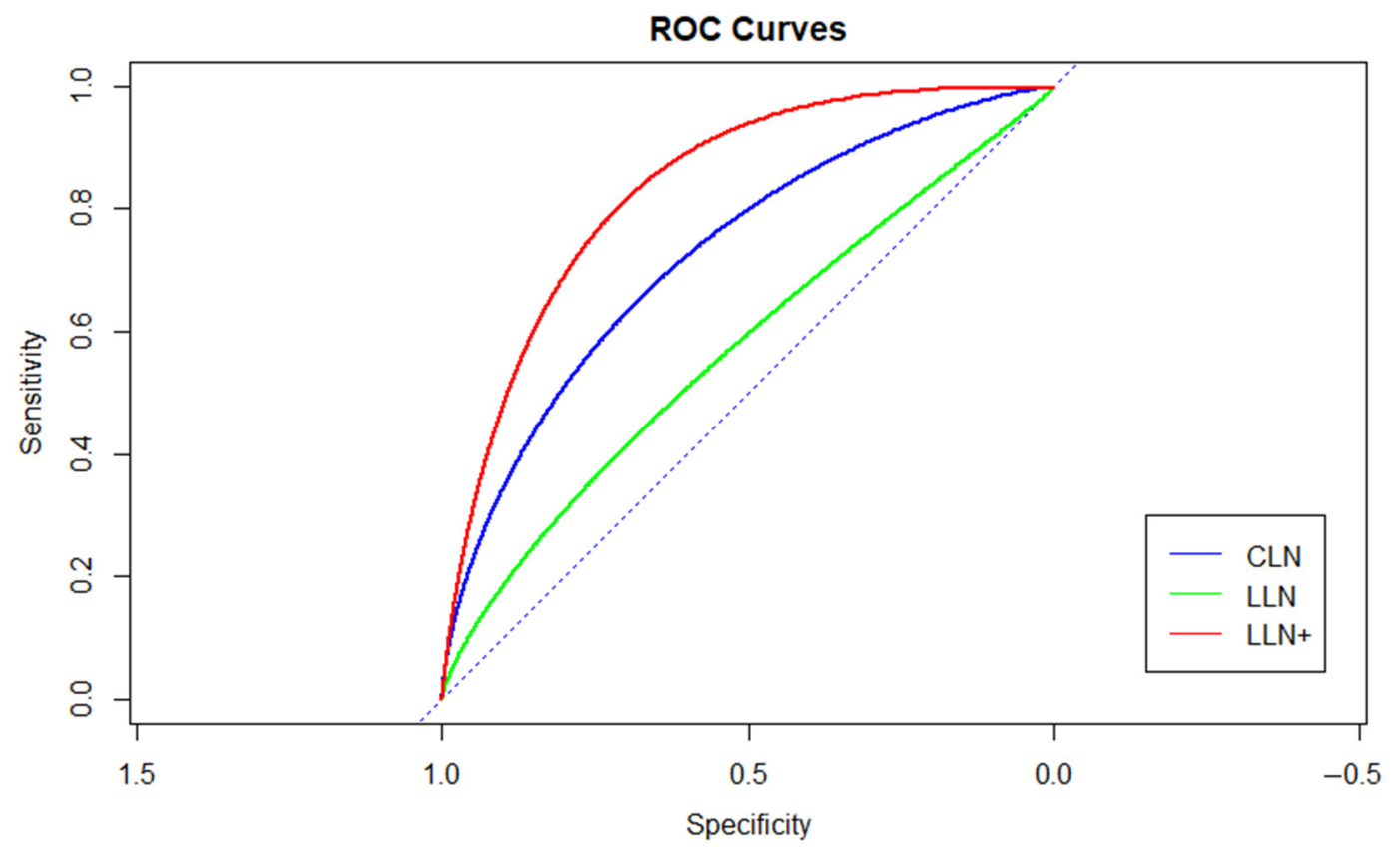

Figure 1. The number of LLN is a poor predictor of $C L N+\geq 1$.

\subsection{Multivariate Analysis}

The multivariate analysis (Table 2) revealed that the best-fitting model according to AIC (=67.98) included the following variables: Age, Sex, Localization, CLN $>7$ and LLN $+<4$. The model shows that center-lobar cancer localization and LLN $+<4$ appear to be protective values (CLN+ $=0$ and no recurrence) with ORs of $0.00595 \% \mathrm{IC}$ $\left(8.12 \times 10^{-5}-8.21 \times 10^{-2}\right) \quad(p$-value $<0.05)$ and $0.02095 \%$ IC $\left(7.81 \times 10^{-4}-0.164\right)$ $(p$-value $<0.05)$, respectively. 
Table 1. The number of CLN and LLN+ were found to be a moderately accurate predictor of CLN+ $\geq 1$. TIRADS Classification: TIRADS I: Normal thyroid US; TIRADS II: Benign Aspects; TIRADS III: Probably Benign Aspects; TIRADS IV A Low Suspicious Aspect; TIRADS IVB 1 or 2 signs of High suspicious aspects and no Adenopathy; TIRADS 5: $\geq 3$ of High Suspicious aspects and/or Adenopathy. The Bethesda System for Reporting Thyroid Cytopathology: Bethesda I: Nondiagnostic or Unsatisfactory; Bethesda II: Benign; Bethesda III: Atypia of undetermined significance or follicular lesion of undetermined significance; Bethesda IV: Follicular neoplasm or suspicious for a follicular neoplasm; Bethesda V: Suspicious for malignancy; Bethesda VI: Malignant. Nodule location: Site 1: upper lobe pole; Site 2: middle lobe; Site 3: isthmic, paraisthmic or lower pole $[19,20]$.

\begin{tabular}{|c|c|c|c|c|c|}
\hline Variable & CLN+ 0_Recovery & CLN $+\geq 1$ & Total & OR $(95 \% \mathrm{CI})$ & $p$-Value \\
\hline Age & 47 & 46 & & & 0.677 \\
\hline Sex $M$ & 6 & 22 & 28 & \multirow{3}{*}{$\begin{array}{c}2.77 \\
(0.92-9.60)\end{array}$} & \multirow{3}{*}{0.05849} \\
\hline Sex F & 26 & 34 & 60 & & \\
\hline Total & 32 & 56 & 88 & & \\
\hline TSH & 2.19 & 2.15 & & & 0.5318 \\
\hline $\begin{array}{c}\text { Autoimmunity } \\
\text { YES }\end{array}$ & 7 & 12 & 19 & \multirow{3}{*}{$\begin{array}{c}0.97 \\
(0.31-3.32)\end{array}$} & \multirow{3}{*}{0.9999} \\
\hline Autoimmunity NO & 25 & 44 & 69 & & \\
\hline Total & 32 & 56 & 88 & & \\
\hline Size 1 & 27 & 44 & 71 & & \multirow{4}{*}{0.8454} \\
\hline Size 2 & 2 & 6 & 8 & & \\
\hline Size 3 & 3 & 6 & 9 & & \\
\hline Total & 32 & 56 & 88 & & \\
\hline Site 1 & 12 & 31 & 43 & & \multirow{4}{*}{$8.141 \times 10^{-6}$} \\
\hline Site 2 & 13 & 1 & 14 & & \\
\hline Site 3 & 7 & 24 & 31 & & \\
\hline Total & 32 & 56 & 88 & & \\
\hline Multifocality NO & 40 & 9 & 49 & \multirow{3}{*}{$\begin{array}{c}0.37 \\
(0.06-1.65)\end{array}$} & \multirow{3}{*}{0.2136} \\
\hline Multifocality YES & 36 & 3 & 39 & & \\
\hline Total & 32 & 56 & 88 & & \\
\hline Bethesda 1 & 1 & 1 & 2 & & \multirow{7}{*}{0.3606} \\
\hline Bethesda 2 & 3 & 4 & 7 & & \\
\hline Bethesda 3 & 9 & 11 & 20 & & \\
\hline Bethesda 4 & 15 & 22 & 37 & & \\
\hline Bethesda 5 & 4 & 13 & 17 & & \\
\hline Bethesda 6 & 0 & 5 & 5 & & \\
\hline Total & 32 & 56 & 88 & & \\
\hline TIRADS 3 & 2 & 1 & 3 & & \multirow{4}{*}{0.008372} \\
\hline TIRADS 4 & 14 & 10 & 24 & & \\
\hline TIRADS 5 & 16 & 45 & 61 & & \\
\hline Total & 32 & 56 & 88 & & \\
\hline CLN & 4.4 & 7.5 & & & 0.0008545 \\
\hline LLN & 17.31 & 19.57 & & & 0.3917 \\
\hline LLN+ & 1.81 & 4.36 & & & $5.741 \times 10^{-7}$ \\
\hline
\end{tabular}

ROC Curves. Bold format of data mean $p$-Values $<0.05$.

From the analysis of Kaplan-Meier curves (Figure 2), it can be seen that the difference in disease-free survival between the group of patients in which no central lymph nodes were found and the group with metastatic central lymph nodes was significant $(p<0.05)$.

Finally, we found it interesting to note that patients with CLN $=0$ had a small number of metastatic LLN (4 or less) and none of them experienced persistence or disease recurrence.

Variables found to be non-significant in the univariate analysis (age, sex, TSH levels, autoimmunity, multifocality, Bethesda category, TIRADS category) were not considered in the multivariate analysis.

Variables found. 
Table 2. The model shows that center-lobar cancer localization and LLN $+<4$ appear to be protective values.

\begin{tabular}{ccccc}
\hline Variable & OR & IC (Inf) $\mathbf{9 5 \%}$ & IC (Sup) $\mathbf{9 5 \%}$ & $p$-Value \\
\hline Age & 0.959 & 0.907 & 1.007 & 0.10725 \\
Sex & 0.564 & 0.073 & 3.448 & 0.54998 \\
Site 2 & 0.005 & $8.12 \mathrm{e}^{-5}$ & $8.21 \mathrm{e}^{-2}$ & $0.00210^{* *}$ \\
Site 3 & 1.224 & 0.275 & 5.491 & 0.78752 \\
CLN $>7$ & 1.587 & 1.231 & 2.195 & $0.00149^{* *}$ \\
LLN+<4 & 0.020 & $7.81 \mathrm{e}^{-4}$ & 0.164 & $0.00238^{* *}$ \\
\hline
\end{tabular}

${ }^{* *}$ mean $p$-values lower than 0.05 .

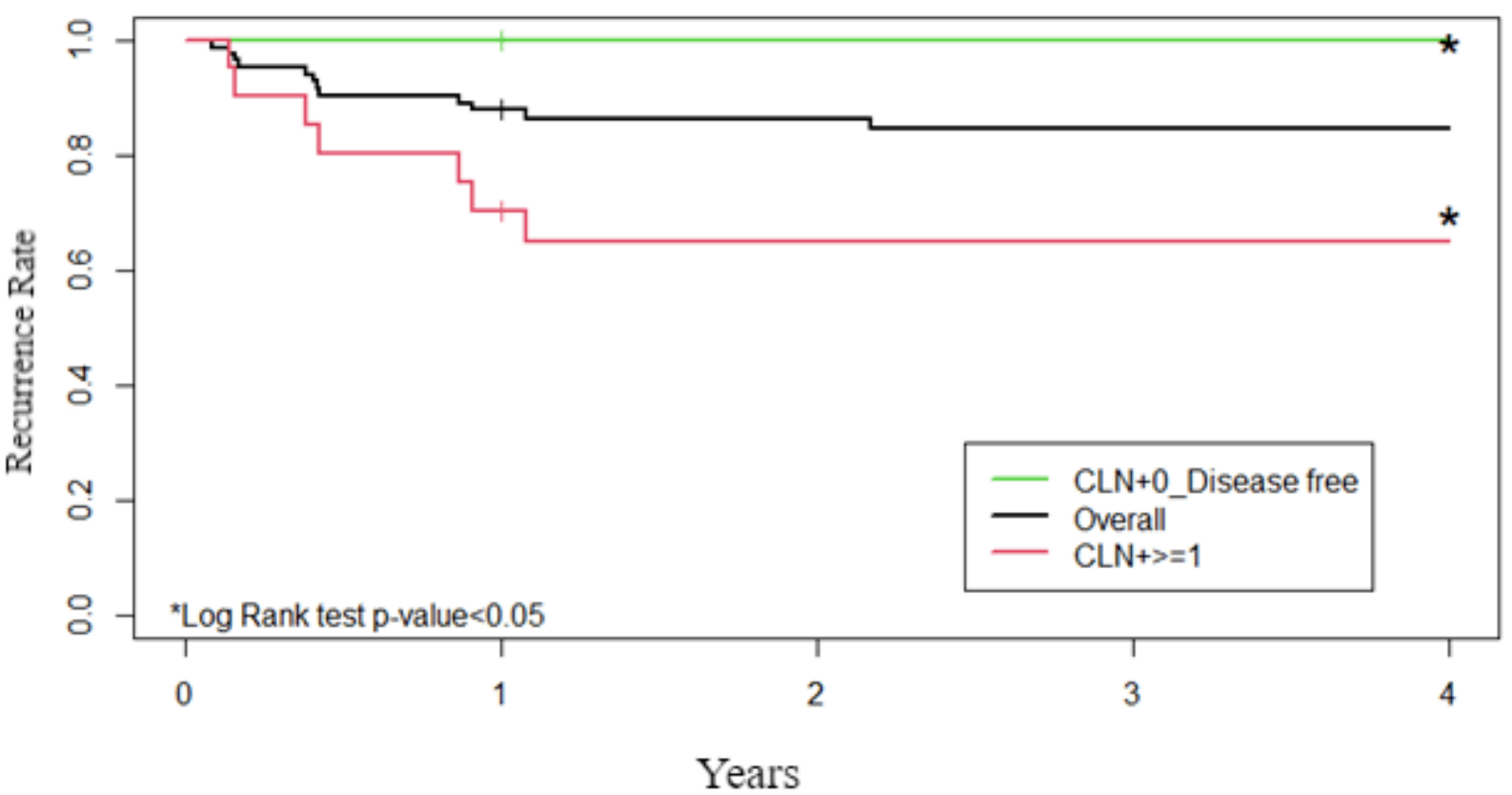

Figure 2. The group of patients with no central lymph nodes involvement has longer disease-free survival than the group with metastatic central lymph nodes $(p<0.05)$. Kaplan-Meier Curves.

\section{Discussion}

Nowadays, the role of PCND in PTC is still debated, with studies proposing to avoid this procedure for early stages and limit CND to cases of clinically positive central lymph nodes [21,22] and others showing benefits even for stages below T3 [23-25]. Given the advantages, especially from the point of view of postoperative radiometabolic treatment, and possible complications such as hypoparathyroidism and recurrent nerve palsy, careful case selection and consensus in the context of the multidisciplinary team has been proposed by others [26]. In any case, the position that PCND does not change the overall survival rate of PTC seems to be prevalent [27-29].

Currently, the ATA, CCN and ESES guidelines consider the presence of lateral neck metastases as an indication for CND [4,6,30]. This is also supported by the order of the lymphatic diffusion pathways, which, however, may vary for portions of thyroid tissue located near the upper poles of the gland, as stated in several studies [13,14,31-33]. This was also confirmed in a detailed anatomical study [34].

Central lymph node metastases in PTC are extremely frequent (up to $80 \%$ ), but most of them are microscopic, detected after careful histopathological evaluation and have no clinical significance $[2,16,35]$. On the other hand, in studies considering prophylactic lateral cervical dissection, the occurrence of metastases in levels II-V was well above 50\% [36], although there is very little support for such a choice and not much data available on the subject. These data have to be compared with those related to the risk of PTC recurrence, which is very low in the most common variants of PTC and, in any case, ranging from 
$1 \%$ to $40 \%$ [16,37], and, in an even clearer perspective, in the 5-year disease-free survival, which reaches $98 \%$ [4]. This debate could be concluded by stating that, obviously within certain limits, there is no correlation between central or lateral lymph node metastases, rate of locoregional recurrence and in general the prognosis of PTC $[4,16,21,38]$. These limits may be constituted by the real numerical and especially volumetric consistency of the affected lymph nodes [16], as well as by the prognostic factors of cancer, which, however, most likely act independently from lymph node metastases [39].

The attitude taken at our institution with regard to PCND has gone from a gradual enthusiasm that had extended its indications also to early stages of PTC (T2 and sometimes T1), which culminated in the middle of the last decade $[9,24]$, to a gradual alignment with the actual indications of the main scientific societies mentioned above, so that, in fact, we now perform PCND only in T3 and in N1b with any T.

In this study, we found a relapse rate of $13.7 \%(12)$, similar to percentage described in several other studies $[40,41]$.

The number of patients with positive LLN but without central compartment involvement was very high $(32=36.4 \%)$. This high prevalence of LLN+ without CLN+ would be sufficient to justify further investigation, which led to our results.

An aspect that we found interesting in our study was the finding that the presence of few positive LLNs $(<4)$ seems to be protective compared to the presence of CLN metastases; therefore, in such circumstances, the presence of a sporadic metastasis in the lateral site might be predictive of skip metastasis. A finding that could be investigated in the future is the association of more advanced TIRADS with the increased likelihood of central metastases in the presence of known LLN metastases. Although we are not able to give a definite interpretation to this statistical finding, we believe we can explain this effect by a probably greater aggressiveness of the tumor that could tend to be associated with more advanced morphological aspects. A similar association was not found when evaluating cytological features with the Bethesda system, whose degree of alterations do not correlate in any way (indeed we did not expect it) with a more extensive metastatic spread of the tumor.

The first interesting result of our study is evidenced by the multivariate analysis, which confirms the importance of the apical location in the thyroid lobe as a risk factor for the presence of possible skip metastases and, conversely, a certain "protective" role of cancer locations other than this one in the context of the thyroid lobe.

Another attractive finding arising from the results of the Kaplan-Meier curves is the better disease-free survival reported in the group of patients without involvement of CLN, even with LLN+, demonstrating a role of the CLN- as a positive prognostic factor in terms of persistence or recurrence. Conversely, the simultaneous presence of CLN+ and LLN+ entails in a worse prognosis. All these results could constitute a further justification to limit prophylactic CND in the presence of isolated skip metastases, provided that these do not hide central metastases [40].

We are aware of several biases in our study: firstly, its retrospective nature; secondly, the expertise-dependence of lymph node clearing during lymph node dissections; and thirdly, we consider the study to be very large, with follow-up ranging from 6 months to 10 years. Furthermore, the empiricism with which recurrence was established and with which, in turn, it is distanced from persistence. Furthermore, the enrolment of laterocervical dissections less extensive than II-V may constitute a bias but this type of surgical choice is justified and validated in the literature [41-43].

Another bias is the lack of evaluation of some prognostic factors, such as angioinvasiveness, extracapsular or extranodal extension, but usually, such evaluations are not reliably answered during the preoperative workup and, on the other hand, our intention was to find reliable answers to the question "what to do if a certain condition is found" in a real scenario. Finally, we did not compare a population of patients who underwent CLND plus LLND versus only LLND. In this perspective, the comparison between the two groups should be considered virtual. 


\section{Conclusions}

At present, we cannot consider the data obtained from this study sufficient to lead to the adoption of new treatment protocols.

Far from providing definitive data on the existence of a category of PTC patients in whom level VI dissection can be avoided even in the presence of lateral cervical metastases, this study aims to provide further food for thought and add data to achieve the goal of avoiding central compartment dissection, albeit in a restricted category of patients, and thus limiting risks and sequelae of this not entirely harmless procedure.

Author Contributions: Conceptualization, G.G., C.C. and G.S.; Data curation, S.M., I.V., M.P.P., C.A., F.S., E.M.R. and A.C.; Formal analysis, S.M.; Investigation, G.O., G.C. and G.M.; Methodology, G.G., G.O., G.C., G.M., C.C. and G.S.; Software, A.C.; Supervision, G.S.; Validation, C.C. and G.S.; Writing-original draft, G.G.; Writing-review \& editing, G.S. All authors have read and agreed to the published version of the manuscript.

Funding: This research received no external funding.

Institutional Review Board Statement: The study was conducted according to the guidelines of the Declaration of Helsinki and Ethics review and approval for this study was waived because of our institution's nonrequirement for retrospective studies.

Informed Consent Statement: Informed consent was obtained from all subjects involved in the study. This retrospective study analyzes anonymized data already in institutional database.

Data Availability Statement: Digital and paper archives of the Department of Surgical Oncological and Oral Sciences, Policlinico P. Giaccone, University of Palermo, Via L Giuffré, 5, 90127 Palermo, Italy.

Conflicts of Interest: The authors declare no conflict of interest.

\section{References}

1. Eltelety, A.M.; Terris, D.J. Neck Dissection in the Surgical Treatment of Thyroid Cancer. Endocrinol. Metab. Clin. N. Am. 2019, 48, 143-151. [CrossRef] [PubMed]

2. Thompson, A.M.; Turner, R.M.; Hayen, A.; Aniss, A.; Jalaty, S.; Learoyd, D.L.; Sidhu, S.; Delbridge, L.; Yeh, M.W.; Clifton-Bligh, R.; et al. A preoperative nomogram for the prediction of ipsilateral central compartment lymph node metastases in papillary thyroid cancer. Thyroid 2014, 24, 675-682. [CrossRef]

3. Sakorafas, G.H.; Koureas, A.; Mpampali, I.; Balalis, D.; Nasikas, D.; Ganztzoulas, S. Patterns of Lymph Node Metastasis in Differentiated Thyroid Cancer; Clinical Implications with Particular Emphasis on the Emerging Role of Compartment-Oriented Lymph Node Dissection. Oncol. Res. Treat. 2019, 42, 143-147. [CrossRef] [PubMed]

4. Haugen, B.R.; Alexander, E.K.; Bible, K.C.; Doherty, G.M.; Mandel, S.J.; Nikiforov, Y.E.; Pacini, F.; Randolph, G.W.; Sawka, A.M.; Schlumberger, M.; et al. 2015 American Thyroid Association Management Guidelines for Adult Patients with Thyroid Nodules and Differentiated Thyroid Cancer: The American Thyroid Association Guidelines Task Force on Thyroid Nodules and Differentiated Thyroid Cancer. Thyroid 2016, 26, 1-133. [CrossRef] [PubMed]

5. Doubleday, A.; Sippel, R.S. Surgical options for thyroid cancer and post-surgical management. Expert Rev. Endocrinol. Metab. 2018, 13, 137-148. [CrossRef] [PubMed]

6. Sancho, J.J.; Lennard, T.W.J.; Paunovic, I.; Triponez, F.; Sitges-Serra, A. Prophylactic central neck disection in papillary thyroid cancer: A consensus report of the European Society of Endocrine Surgeons (ESES). Langenbeck's Arch. Surg. 2014, 399, 155-163. [CrossRef] [PubMed]

7. Palestini, N.; Borasi, A.; Cestino, L.; Freddi, M.; Odasso, C.; Robecchi, A. Is central neck dissection a safe procedure in the treatment of papillary thyroid cancer? Our experience. Langenbeck's Arch. Surg. 2008, 393, 693-698. [CrossRef] [PubMed]

8. Mazzaferri, E.L.; Doherty, G.M.; Steward, D.L. The pros and cons of prophylactic central compartment lymph node dissection for papillary thyroid carcinoma. Thyroid 2009, 19, 683-689. [CrossRef] [PubMed]

9. Scerrino, G.; Di Giovanni, S.; Salamone, G.; Melfa, G.I.; Inviati, A.; Raspanti, C.; Gulotta, G. Surgical complications in prophylactic central neck dissection: Preliminary findings from a retrospective cohort study. Minerva Chir. 2014, 69 (Suppl. S1), $131-134$.

10. Lombardi, D.; Accorona, R.; Paderno, A.; Cappelli, C.; Nicolai, P. Morbidity of central neck dissection for papillary thyroid cancer. Gland. Surg. 2017, 6, 492-500. [CrossRef]

11. Calò, P.; Conzo, G.; Raffaelli, M.; Medas, F.; Gambardella, C.; De Crea, C.; Gordini, L.; Patrone, R.; Sessa, L.; Erdas, E.; et al. Total thyroidectomy alone versus ipsilateral versus bilateral prophylactic central neck dissection in clinically node-negative differentiated thyroid carcinoma. A retrospective multicenter study. Eur. J. Surg. Oncol. EJSO 2017, 43, 126-132. [CrossRef]

12. Liu, X.; Zhang, D.; Zhang, G.; Zhao, L.; Zhou, L.; Fu, Y.; Li, S.; Zhao, Y.; Li, C.; Wu, C.-W.; et al. Laryngeal nerve morbidity in 1.273 central node dissections for thyroid cancer. Surg. Oncol. 2018, 27, A21-A25. [CrossRef] [PubMed] 
13. Lee, Y.S.; Shin, S.-C.; Lim, Y.-S.; Lee, J.-C.; Wang, S.-G.; Son, S.-M.; Kim, I.-J.; Lee, B.-J. Tumor location-dependent skip lateral cervical lymph node metastasis in papillary thyroid cancer. Head Neck 2014, 36, 887-891. [CrossRef] [PubMed]

14. Attard, A.; Paladino, N.C.; Monte, A.I.L.; Falco, N.; Melfa, G.; Rotolo, G.; Rizzuto, S.; Gulotta, E.; Salamone, G.; Bonventre, S.; et al. Skip metastases to lateral cervical lymph nodes in differentiated thyroid cancer: A systematic review. BMC Surg. 2019, 18 (Suppl. S1), 112. [CrossRef] [PubMed]

15. Hu, D.; Lin, H.; Zeng, X.; Wang, T.; Deng, J.; Su, X. Risk Factors for and Prediction Model of Skip Metastasis to Lateral Lymph Nodes in Papillary Thyroid Carcinoma. World J. Surg. 2019, 44, 1498-1505. [CrossRef]

16. Harries, V.; BS, M.M.; Wang, L.Y.; Tuttle, R.M.; Wong, R.J.; Shaha, A.R.; Shah, J.P.; Patel, S.G.; Ganly, I. Is a Prophylactic Central Compartment Neck Dissection Required in Papillary Thyroid Carcinoma Patients with Clinically Involved Lateral Compartment Lymph Nodes? Ann. Surg. Oncol. 2021, 28, 512-518. [CrossRef] [PubMed]

17. Melfa, G.; Porrello, C.; Cocorullo, G.; Raspanti, C.; Rotolo, G.; Attard, A.; Gullo, R.; Bonventre, S.; Gulotta, G.; Scerrino, G. Surgeon volume and hospital volume in endocrine neck surgery: How many procedures are needed for reaching a safety level and acceptable costs? A systematic narrative review. Il G. Di Chir. 2018, 39, 5-11. [CrossRef] [PubMed]

18. Lorenz, K.; Raffaeli, M.; Barczyński, M.; Lorente-Poch, L.; Sancho, J. Volume, outcomes, and quality standards in thyroid surgery: An evidence-based analysis-European Society of Endocrine Surgeons (ESES) positional statement. Langenbeck's Arch. Surg. 2020, 405, 401-425. [CrossRef]

19. Cibas, E.S.; Ali, S.Z. The 2017 Bethesda System for Reporting Thyroid Cytopathology. Thyroid 2017, 27, 1341-1346. [CrossRef]

20. Grant, E.G.; Tessler, F.N.; Hoang, J.K.; Langer, J.E.; Beland, M.D.; Berland, L.L.; Cronan, J.J.; Desser, T.S.; Frates, M.C.; Hamper, U.M.; et al. Thyroid Ultrasound Reporting Lexicon: White Paper of the ACR Thyroid Imaging, Reporting and Data System (TIRADS) Committee. J. Am. Coll. Radiol. 2015, 12, 1272-1279. [CrossRef]

21. Sippel, R.S.; Robbins, S.E.; Poehls, J.L.; Pitt, S.C.; Chen, H.; Leverson, G.; Long, K.L.; Schneider, D.F.; Connor, N.P. A randomized controlled clinical trial: No clear benefit to prophylactic central neck dissection in patients with clinically node negative papillary thyroid cancer. Ann. Surg. 2020, 272, 496-503. [CrossRef] [PubMed]

22. Gambardella, C.; Tartaglia, E.; Nunziata, A.; Izzo, G.; Siciliano, G.; Cavallo, F.; Mauriello, C.; Napolitano, S.; Thomas, G.; Testa, D.; et al. Clinical significance of prophylactic central compartment neck dissection in the treatment of clinically node-negative papillary thyroid cancer patients. World J. Surg. Oncol. 2016, 14, 1-5. [CrossRef] [PubMed]

23. Barczyński, M.; Konturek, A.; Stopa, M.; Nowak, W. Prophylactic central neck dissection for papillary thyroid cancer. BJS 2013, 100, 410-418. [CrossRef] [PubMed]

24. Scerrino, G.; Attard, A.; Melfa, G.; Raspanti, C.; Di Giovanni, S.; Attard, M.; Inviati, A.; Mazzola, S.; Modica, G.; Gulotta, G.; et al Role of prophylactic central neck dissection in cN0-papillary thyroid carcinoma: Results from a high-prevalence area. Minerva Chir. 2015, 71, 159-167.

25. Yazıcı, D.; Çolakoğlu, B.; Sağlam, B.; Sezer, H.; Kapran, Y.; Aydın, Ö.; Demirkol, M.O.; Alagöl, F.; Terzioğlu, T. Effect of prophylactic central neck dissection on the surgical outcomes in papillary thyroid cancer: Experience in a single center. Eur. Arch. Oto-Rhino-Laryngol. 2020, 277, 1491-1497. [CrossRef]

26. Chen, L.; Wu, Y.-H.; Lee, C.-H.; Chen, H.-A.; Loh, E.-W.; Tam, K.-W. Prophylactic Central Neck Dissection for Papillary Thyroid Carcinoma with Clinically Uninvolved Central Neck Lymph Nodes: A Systematic Review and Meta-analysis. World J. Surg. 2018, 42, 2846-2857. [CrossRef]

27. Leboulleux, S.; Rubino, C.; Baudin, E.; Caillou, B.; Hartl, D.M.; Bidart, J.M.; Travagli, J.P.; Schlumberger, M. Prognostic factors for persistent or recurrent disease of papillary thyroid carcinoma with neck lymph node metastases and/or tumor extension beyond the thyroid capsule at initial diagnosis. J. Clin. Endocrinol. Metab. 2005, 90, 5723-5729. [CrossRef]

28. Toniato, A.; Boschin, I.M.; Casara, D.; Mazzarotto, R.; Rubello, D.; Pelizzo, M. Papillary Thyroid Carcinoma: Factors Influencing Recurrence and Survival. Ann. Surg. Oncol. 2008, 15, 1518-1522. [CrossRef]

29. Bardet, S.; Malville, E.; Rame, J.P.; Babin, E.; Samama, G.; De Raucourt, D.; Michels, J.J.; Reznik, Y.; Henry-Amar, M. Macroscopic lymph-node involvement and neck dissection predict lymph-node recurrence in papillary thyroid carcinoma. Eur. J. Endocrinol. 2008, 158, 551-560. [CrossRef]

30. National Comprehensive Cancer Network. NCCN Clinicial Practice Guidelines in Oncology-Thyroid Carcinoma. Available online: https: / / www.nccn.org/ (accessed on 20 April 2019).

31. Zhang, L.; Wei, W.-J.; Ji, Q.-H.; Zhu, Y.-X.; Wang, Z.-Y.; Wang, Y.; Huang, C.-P.; Shen, Q.; Li, D.-S.; Wu, Y. Risk Factors for Neck Nodal Metastasis in Papillary Thyroid Microcarcinoma: A Study of 1066 Patients. J. Clin. Endocrinol. Metab. 2012, 97, 1250-1257. [CrossRef]

32. Xiang, D.; Xie, L.; Xu, Y.; Li, Z.; Hong, Y.; Wang, P. Papillary thyroid microcarcinomas located at the middle part of the middle third of the thyroid gland correlates with the presence of neck metastasis. Surgery 2015, 157, 526-533. [CrossRef]

33. Zhan, X.; Xue, S.; Yin, Y.; Chen, G. Related factors on skip metastasis of neck lymph node in papillary thyroid carcinoma. Zhonghua Wai Ke Za Zhi Chin. J. Surg. 2017, 55, 599-602. [PubMed]

34. Likhterov, I.; Dos Reis, L.L.; Urken, M.L. Central compartment management in patients with papillary thyroid cancer presenting with metastatic disease to the lateral neck: Anatomic pathways of lymphatic spread. Head Neck 2017, 39, 853-859. [CrossRef] [PubMed] 
35. Randolph, G.W.; Duh, Q.Y.; Heller, K.S.; LiVolsi, V.A.; Mandel, S.J.; Steward, D.L.; Tufano, R.P.; Tuttle, R.M. The prognostic significance of nodal metastases from papillary thyroid carcinoma can be stratified based on the size and number of metastatic lymph nodes, as well as the presence of extranodal extension. Thyroid 2012, 22, 1144-1152. [CrossRef] [PubMed]

36. Mulla, M.G.; Knoefel, W.T.; Gilbert, J.; McGregor, A.; Schulte, K.M. Lateral cervical lymph node metastases in papillary thyroid cancer: A systematic review of imaging-guided and prophylactic removal of the lateral compartment. Clin. Endocrinol. 2012, 77, 126-131. [CrossRef]

37. National Cancer Institute. Surveillance, Epidemiology, and End Results Program (SEER): Thyroid Cancer. Available online: https: / / seer.cancer.gov/statfacts/html/thyro.html (accessed on 30 June 2021).

38. Viola, D.; Materazzi, G.; Valerio, L.; Molinaro, E.; Agate, L.; Faviana, P.; Seccia, V.; Sensi, E.; Romei, C.; Piaggi, P.; et al. Prophylactic central compartment lymph node dissection in papillary thyroid carci-noma: Clinical implications derived from the first prospective randomized controlled single institution study. J. Clin. Endocrinol. Metab. 2015, 100, 1316-1324. [CrossRef]

39. Cipriani, N.A. Prognostic Parameters in Differentiated Thyroid Carcinomas. Surg. Pathol. Clin. 2019, 12, 883-900. [CrossRef]

40. Lee, D.Y.; Oh, K.H.; Cho, J.-G.; Kwon, S.-Y.; Woo, J.-S.; Baek, S.-K.; Jung, K.-Y. The Benefits and Risks of Prophylactic Central Neck Dissection for Papillary Thyroid Carcinoma: Prospective Cohort Study. Int. J. Endocrinol. 2015, 2015, 571480. [CrossRef]

41. Chéreau, N.; Buffet, C.; Trésallet, C.; Tissier, F.; Leenhardt, L.; Menegaux, F. Recurrence of papillary thyroid carcinoma with lateral cervical node metastases: Predictive factors and operative management. Surgery 2016, 159, 755-762. [CrossRef]

42. Chinn, S.; Zafereo, M.; Waguespack, S.G.; Edeiken, B.S.; Roberts, D.B.; Clayman, G.L. Long-Term Outcomes of Lateral Neck Dissection in Patients with Recurrent or Persistent Well-Differentiated Thyroid Cancer. Thyroid 2017, 27, 1291-1299. [CrossRef]

43. Hartl, D.M.; Al Ghuzlan, A.; Borget, I.; Leboulleux, S.; Mirghani, H.; Schlumberger, M. Prophylactic Level II Neck Dissection Guided by Frozen Section for Clinically Node-Negative Papillary Thyroid Carcinoma: Is It Useful? World J. Surg. 2013, 38, 667-672. [CrossRef] [PubMed] 